\title{
The role of endocannabinoids in visceral hyposensitivity induced by rapid eye movement sleep deprivation in rats: Regional differences
}

\author{
LIYUN WANG* ${ }^{*}$, TING YANG*, WEI QIAN and XIAOHUA HOU \\ Division of Gastroenterology, Union Hospital of Tongji Medical College, \\ Huazhong University of Science and Technology, Wuhan 430022, P.R. China
}

Received July 23, 2010; Accepted September 20, 2010

DOI: $10.3892 / \mathrm{ijmm} .2010 .547$

\begin{abstract}
Visceral hypersensitivity is one of the most important mechanisms of functional gastrointestinal diseases. Our previous studies have shown that rapid eye movement (REM) sleep deprivation (REMSD) decreases visceral sensitivity in rats, but the mechanisms involved in this effect, have not yet been clarified. In this study, we investigated the role of the CNS and peripheral endocannabinoids in visceral hyposensitivity induced by REMSD. Animals were randomly divided into the cage-yoked (YC), the REMSD group, which suffered from REMSD for $48 \mathrm{~h}$, and the group with the interventions of Rimonabant after REMSD. The visceral sensitivity of all the groups was assessed, and the expressions of cannabinoid receptor (CB1R), fatty acid amide hydrolase (FAAH) and monoacylglycerol lipase (MGL) in the CNS and gut regions, were detected. We demonstrate that REMSD decreases visceral sensitivity in rats and that the Rimonabant intervention antagonizes this effect. The expression of CB1R in the CNS region was significantly higher in the REMSD compared to the YC group. We did not see similar results in the gut. At the same time, the expressions of FAAH and MGL in the CNS and colon, excluding the iliac terminus, were lower in the REMSD compared to the YC group. Endocannabinoids are involved in the mechanism of visceral hyposensitivity in rats induced by REMSD. Possibly those in the CNS play the main role in this activity.
\end{abstract}

\section{Introduction}

Visceral hypersensitivity has been shown to be a biological marker in many patients with functional gastrointestinal

Correspondence to: Professor Xiaohua Hou, Union Hospital of Tongji Medical College, Huazhong University of Science and Technology, 1277 Jiefang Avenue, Wuhan 430022, P.R. China

E-mail: houxh@medmail.com.cn

${ }^{*}$ Contributed equally

Key words: cannabinoid 1 receptor, endocannabinoids, rapid eye movement sleep deprivation, visceral sensitivity diseases, and has attracted increasing attention over the years. However, clinical treatment for visceral hypersensitivity is limited. Methods used to treat visceral hypersensitivity, include the use of antidepressants, anti-anxiety drugs, somatostatin analogs and opioid receptor agonists (1). However, all of these above methods take effect slowly, and are thus slow in relieving symptoms. Rapid eye movement (REM) sleep deprivation (REMSD), which is a hotspot for neuroscience research, is now being used in the treatment of depression (2). Our previous studies have shown that REMSD reduces visceral sensitivity in both normal and highly sensitive rats (3), but the exact mechanisms involved in this effect, are not yet fully understood.

Cannabinoids (CBs), which are derived from the Indian hemp plant, have long been used as medicinal agents as well as recreational drugs. Endocannabinoids, which are endogenously produced messenger molecules that bind to and activate CB receptors in a G-protein-dependent manner (4), show similar pharmacological and physiological characteristics as natural CBs. They mainly consist of two CB receptors, $\mathrm{CB} 1 \mathrm{R}$ and $\mathrm{CB} 2 \mathrm{R}$, and $N$-arachidonoylethanolamide and 2-arachidonoylglycerol (2-AG), are the most abundant endogenous ligands for these receptors. CB2R is almost absent the in brain, and in the CNS, the effect of endocannabinoids is mainly mediated by CB1R (5-7). The peripheral CB1R is located on the neurons of the enteric nervous system, and on the endings of the vagus nerve and spinal sensory neurons, and mediates the antinociceptive and anti-hyperalgesic effects of endocannabinoids, whereas the peripheral CB2R is mainly located in the immune cells $(8,9)$. The enzymes involved in the metabolism of endocannabinoids, include fatty acid amide hydrolase (FAAH) and monoglyceride lipase (MGL). 2-AG is preferentially degraded by MGL, whereas anandamide is hydrolyzed primarily by FAAH $(10,11)$.

There is a close relationship between REM sleep and the endocannabinoid system. Previous studies have shown that the CNS endocannabinoid system is involved in the regulation of sleep and wakefulness (12-14). REMSD increases the activity of the central endocannabinoid system, and leads to the altered expression of CB1R and the enhanced content of 2-AG in the hippocampus (15).

It has been proven that both natural and synthetic cannabis can enhance the pain threshold in animal modes of acute and 
chronic pain, and that this analgesic effect can be antagonized by Rimonabant, a CB1R antagonist. Endocannabinoid receptors are also closely related to visceral pain, and are involved in visceral afferent nerve signals and sensitivity, as well in visceral motor regulation (16). Studies have shown that inflammatory hypersensitivity to colonic distension in rats was abolished by either the CB1R or the CB2R agonist, but only the CB1R antagonist (Rimonabant), which was ineffective in basal conditions, significantly enhanced inflammatory hypersensitivity (17). Therefore, the primary aim of this study was to connect the endocannabinoid system to the altered visceral sensitivity induced by REMSD. We speculate that the endocannabinoid system is involved in the mechanism of visceral hyposensitivity induced by REMSD, and that this involvement occurs mainly through the CB1R pathway.

In order to verify our hypothesis, we carried out an in vivo study using animal models. Rats underwent the process of REMSD by the internationally acknowledged 'flower pot' method, and colorectal distension (CRD) and EMG recordings were used as a measure of visceral sensitivity. We then blocked the activity of the endocannabinoids in the REMSD group by Rimonabant, which is ineffective under normal conditions (17), and observed the alterations in visceral sensitivity in order to confirm the role of endocannabinoids in the mechanism of altered visceral sensitivity after REMSD. In addition, by detecting the different expressions of the $\mathrm{CB}$ receptor, $\mathrm{CB} 1 \mathrm{R}$, and the endocannabinoid metabolic enzymes, FAAH and MGL, in different central and peripheral areas, we could define the location which plays the main role in the endocannabinoid activity.

\section{Materials and methods}

Animals. Twenty-four adult male Sprague-Dawlay rats (experimental ethics approval no. SYXK2004-0028) were obtained from the animal center of Tongji Medical College, Huazhong University of Science and Technology. They weighed between 220-250 g, and were housed in cages in a quiet, warm environment, away from strong light. The 24 male rats were randomly assigned to three groups, including the cage-yoked (YC) control, the REMSD, and the group with the Rimonabant intervention after sleep deprivation. There were 8 rats in each group.

Sleep deprivation. The rats were deprived of REM sleep for $48 \mathrm{~h}$ by the 'flower pot' method (18). In water tanks measuring $30 \times 30 \times 30 \mathrm{~cm}$, the rats were placed on top of platforms $(6 \mathrm{~cm}$ in diameter), surrounded by water, the upper surfaces were only $1 \mathrm{~cm}$ above the water. The rats went through the slow eye movement sleep but they woke up at the REM phase, as the REM sleep-induced decreased muscle tension, caused them to fall off the platforms. In order to avoid disturbance factors in the experiment, the control rats were placed on tanks of similar structure, except that the platforms surrounded by water were $20 \mathrm{~cm}$ in diameter, thus allowing the rats to sleep normally. REMSD started at 6:00 pm and lasted for $48 \mathrm{~h}$. All the rats were sacrificed after $48 \mathrm{~h}$ of REMSD.

After REMSD, the rats in the Rimonabant group received Rimonabant via peritoneal injection (concentration of $1 \mathrm{mg} \mathrm{g}^{-1}$ ).
Ten minutes after the injection, their visceral sensitivity was measured.

Visceral sensitivity measurement. After REMSD for $48 \mathrm{~h}$, CRD and abdominal EMG recordings were carried out simultaneoulsy, reflecting the changes in visceral sensitivity.

$C R D$. CRD was carried out as previoulsy described (19). A polyethylene balloon ( $5 \mathrm{~cm}$ in length, $3 \mathrm{~cm}$ in width) with a connecting scatheless anaesthesia tracheal cannula $(12 \mathrm{Ch} / \mathrm{Fr}$, Euromedical Company, Malaysia) was inserted into the distal colon, $2 \mathrm{~cm}$ from the base of the balloon to the anus. The catheter was fixed to the tail with tape. The CRD procedure consisted of two series of phasic distensions to constant pressures of 20,40, 60 and $80 \mathrm{mmHg}$ (10 sec duration; $40 \mathrm{sec}$ interstimulus interval). The VMR to CRD was quantified by measuring EMG activity in the external oblique muscle. EMG activity was recorded during each pressure condition of the CRD procedure.

EMG recording. EMG recordings were carried out as previously described (20). Two fine-needle electrodes $(0.8 \mathrm{~cm}$ in length) were inserted into the bilateral external oblique muscle at a distance of $1.5 \mathrm{~cm}$ from the middle line, and the limb lead was inserted subcutaneously into the posterior of the right thigh. The leads were connected to the multi-channel physiological signal acquisition aystem (Type RM6240B, Chengdu Instrument factory, China). The equipment was set to the following: High frequency filtering was $50 \mathrm{~Hz}$, low frequency filtering was $3 \mathrm{kHz}$ and the voltage was $1 \mathrm{mV}$. EMG activity which was increased $>100 \mu \mathrm{V}$ above the baseline level was considered to be a meaningful discharge. The discharge frequency of the external oblique muscle under different pressure conditions for $10 \mathrm{sec}$, was measured using matching software. The average of three results was assessed.

Tissue preparation. After CRD, the rats in the control and REMSD groups were sacrificed by decapitation, their brains and intestinal tracts were rapidly removed, and the thalamus, brain stem, spinal cord, distal and proximal colon, and iliac terminus, were dissected on ice and stored at $-80^{\circ} \mathrm{C}$ until use for RT-PCR and Western blotting.

$R T$-PCR. RNA expressions of CB1R, FAAH and MGL in the above-mentioned tissues were detected by RT-PCR. The primers (synthesized by Ying Chum Biological Reagents Co., Shanghai, China) for these genes and B-actin (a 'housekeeping gene' used as the standard) are shown in (Table I).

PCR amplifications were performed using an Advantage cDNA PCR Kit (Promega) with a $20 \mu 1$ final reaction mixture consisting of $2 \mu \mathrm{l}$ cDNA samples, $0.3 \mu 1$ of the primers for each cDNA target, $9.9 \mu 1 \mathrm{ddH}_{2} \mathrm{O}, 12.5 \mu \mathrm{l}$ Advantage cDNA Polymerase Mix and $1 \mu \mathrm{l}$ cDNA PCR reaction buffer. The PCR conditions consisted of initial denaturation at $94^{\circ} \mathrm{C}$ for $5 \mathrm{~min}$, followed by 35 cycles at $94^{\circ} \mathrm{C}$ for $40 \mathrm{sec}, 48^{\circ} \mathrm{C}$ for $40 \mathrm{sec}$, and $72^{\circ} \mathrm{C}$ for $40 \mathrm{sec}$, with the last step being $7 \mathrm{~min}$ of extension at $72^{\circ} \mathrm{C}$.

Western blots. Protein from the above regions was extracted and separated by SDS-polyacrylamide gel electrophoresis, and 
Table I. Primers for the target genes and ß-actin.

\begin{tabular}{|c|c|c|c|}
\hline Target gene & Sequence & Reaction conditions & Product size \\
\hline $\mathrm{CB} 1 \mathrm{R}$ & $\begin{array}{l}\text { F: 5'-CCTGGTTCTGATCCTGGTGG-3' } \\
\text { R: 5'-GTTGTTGGCGTGCTTGTGC-3' }\end{array}$ & $\begin{array}{l}94^{\circ} \mathrm{C} 5 \mathrm{~min} ; 94^{\circ} \mathrm{C} 30 \mathrm{sec}, 5^{\circ} \mathrm{C} 30 \mathrm{sec}, \\
72^{\circ} \mathrm{C} 30 \mathrm{sec}, 35 \mathrm{cycles} ; 72^{\circ} \mathrm{C} 7 \mathrm{~min}\end{array}$ & 289 bp \\
\hline FAAH & $\begin{array}{l}\text { F: 5'-GCCTTTCTCAACAGTATGCG-3' } \\
\text { R: 5'-GATGAACGACCCTCACGAT-3' }\end{array}$ & $\begin{array}{l}94^{\circ} \mathrm{C} 5 \mathrm{~min} ; 94^{\circ} \mathrm{C} 30 \mathrm{sec}, 62^{\circ} \mathrm{C} 30 \mathrm{sec}, \\
72^{\circ} \mathrm{C} 30 \mathrm{sec}, 35 \text { cycles; } 72^{\circ} \mathrm{C} 7 \mathrm{~min}\end{array}$ & 462 bp \\
\hline MGL & $\begin{array}{l}\text { F: 5'-CCAGGCGAACTCCACAGAA-3' } \\
\text { R: 5'-GGAAGTCCGATACCACCAT-3' }\end{array}$ & $\begin{array}{l}94^{\circ} \mathrm{C} 5 \mathrm{~min} ; 94^{\circ} \mathrm{C} 30 \mathrm{sec}, 64^{\circ} \mathrm{C} 30 \mathrm{sec}, \\
72^{\circ} \mathrm{C} 30 \mathrm{sec}, 35 \mathrm{cycles} ; 72^{\circ} \mathrm{C} 7 \mathrm{~min}\end{array}$ & $261 \mathrm{bp}$ \\
\hline ß-actin & $\begin{array}{l}\text { F: 5'AGG GAA ATC GTG CGT GAC-3' } \\
\text { R: 5'ACC CAG GAA GGA AGG GT-3' }\end{array}$ & $\begin{array}{l}94^{\circ} \mathrm{C} 5 \mathrm{~min} ; 94^{\circ} \mathrm{C} 30 \mathrm{sec}, 48^{\circ} \mathrm{C} 30 \mathrm{sec}, \\
72^{\circ} \mathrm{C} 30 \mathrm{sec}, 35 \text { cycles; } 72^{\circ} \mathrm{C} 7 \mathrm{~min}\end{array}$ & $189 \mathrm{bp}$ \\
\hline
\end{tabular}

F, forward; R, reverse.
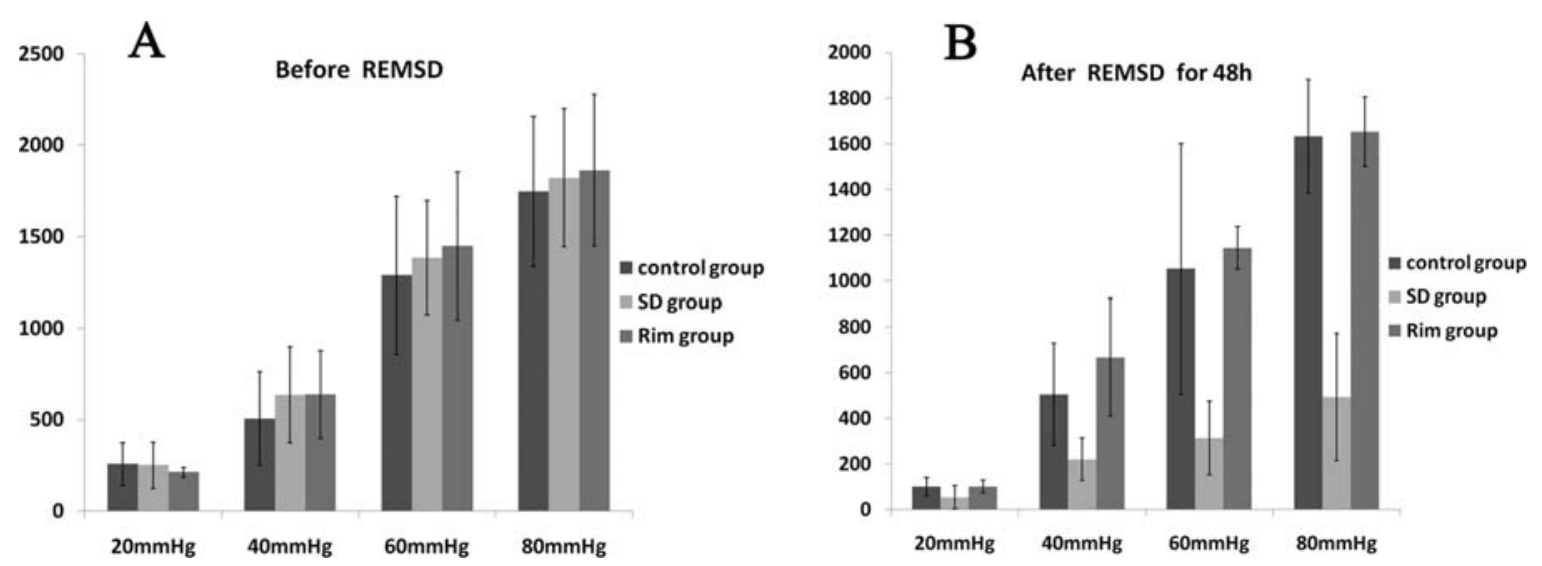

Figure 1. The abdominal wall EMG activities of the rats in each group before REMSD (A), and after REMSD for $48 \mathrm{~h}$ (B). (A) Before REMSD, discharge frequencies of the external oblique muscle under each pressure condition were not significantly different among the three groups. (B) After undergoing REMSD for $48 \mathrm{~h}$, there was a statistically significant difference between the two groups. In the group with the Rimonabant intervention after REMSD for $48 \mathrm{~h}$, the responses to CRD were significantly increased compared to those in the SD group, which showed an increase in discharges of the abdominal oblique muscle. There was no significant difference between the Rim and the control group. (Under the pressure of 20, 40, 60 and $80 \mathrm{mmHg}$, the P-value was 0.069 , 0.016, 0.000 and 0.000, respectively when compared between the control and SD group, and it was $0.991,0.167,0.470$ and 0.862 , respectively when compared between the control and Rim group. When compared between the SD and Rim group, the P-value was $0.681,0.000,0.000$ and 0.000 , respectively). SD group, REMSD group; Rim group, group given Rimonabant.
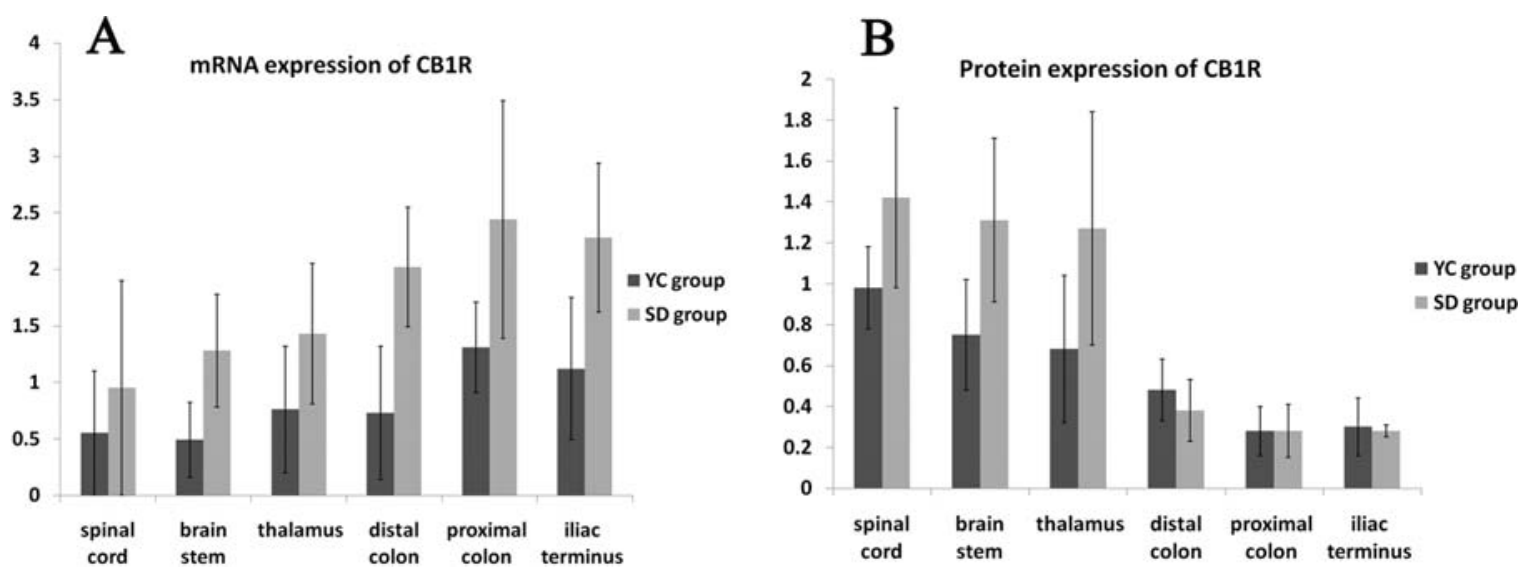

Figure 2. Quantity alteration in mRNA (A) and protein (B) expressions of CB1R in each region after REMSD for $48 \mathrm{~h}$. (A) mRNA expressions of CB1R were significantly increased in the CNS and gut (P-value was $0.028,0.005,0.041,0.001,0.003$ and 0.003 in spinal cord, brain stem, thalamus, distal and proximal colon and iliac terminus, respectively). (B) Protein expressions of CB1R were significantly increased in the CNS (P-value was $0.022,0.006$ and 0.032 in spinal cord, brain stem and thalamus, respectively), but in the gut, there was no significant difference in protein expression between the SD and control group (P-value was $0.145,0.999$ and 0.748 in distal and proximal colon and iliac terminus, respectively). SD group, REMSD group 

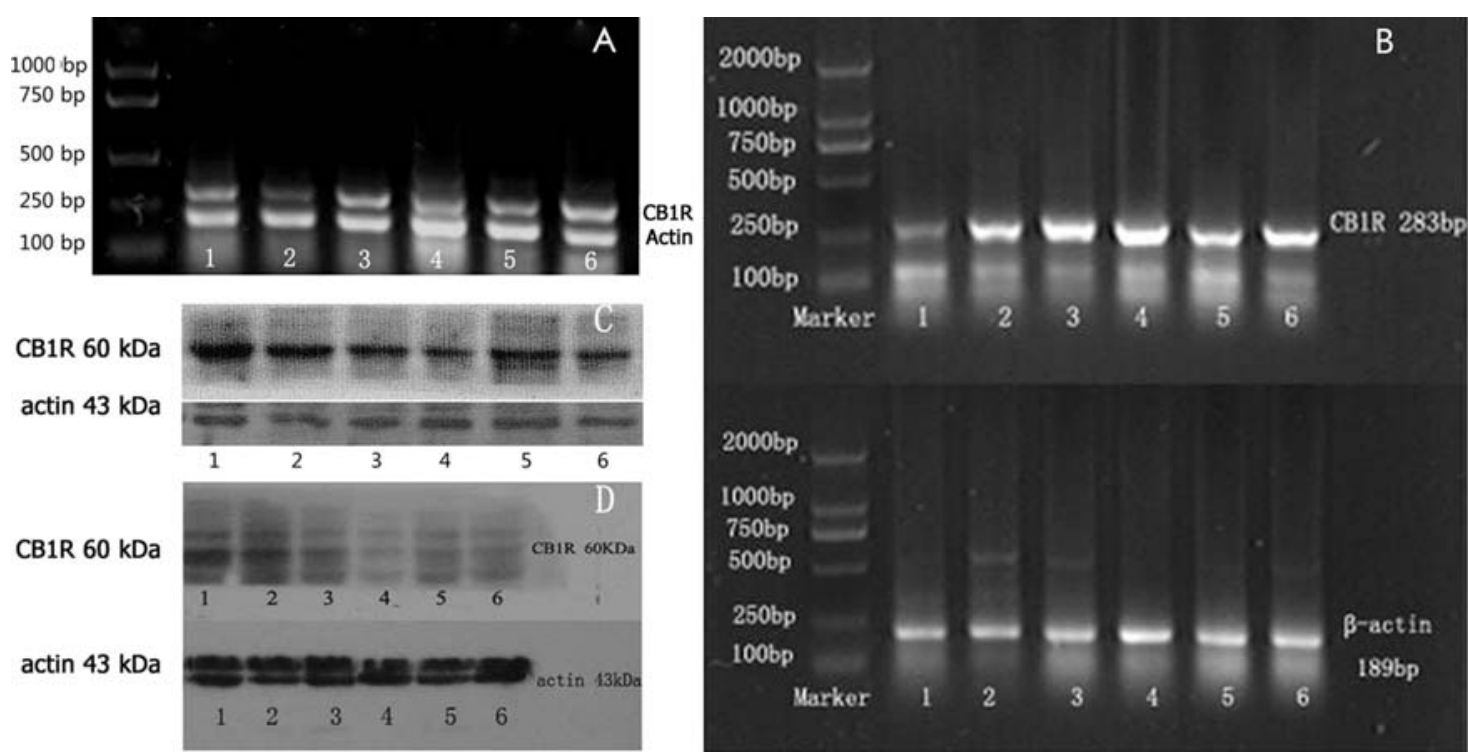

Figure 3. Difference in mRNA (A and B) and protein (C and D) expressions of CB1R in each region after REMSD for 48 h. (A) mRNA expression of CB1R in CNS region. Lane 1, spinal cord of REMSD; 2, spinal cord of YC; 3, brain stem of REMSD; 4, brain stem of YC; 5, thalamus of REMSD; and 6, thalamus of YC group. (B) mRNA expression of CB1R in the gut. Lane 1, distal colon of YC; 2, distal colon of REMSD; 3, proximal colon of YC; 4, proximal colon of REMSD; 5, iliac terminus of YC; and 6, iliac terminus of REMSD group. (C) Protein expression of CB1R in the CNS region. Lane 1, spinal cord of REMSD; 2, spinal cord of YC; 3, brain stem of REMSD; 4, brain stem of YC; 5, thalamus of REMSD; and 6, thalamus of YC group. (D) Protein expression of CB1R in the gut. Lane 1, distal colon of YC; 2, iliac terminus of YC; 3, proximal colon of YC; 4, distal colon of REMSD; 5, iliac terminus of REMSD; and 6, proximal colon of REMSD group.
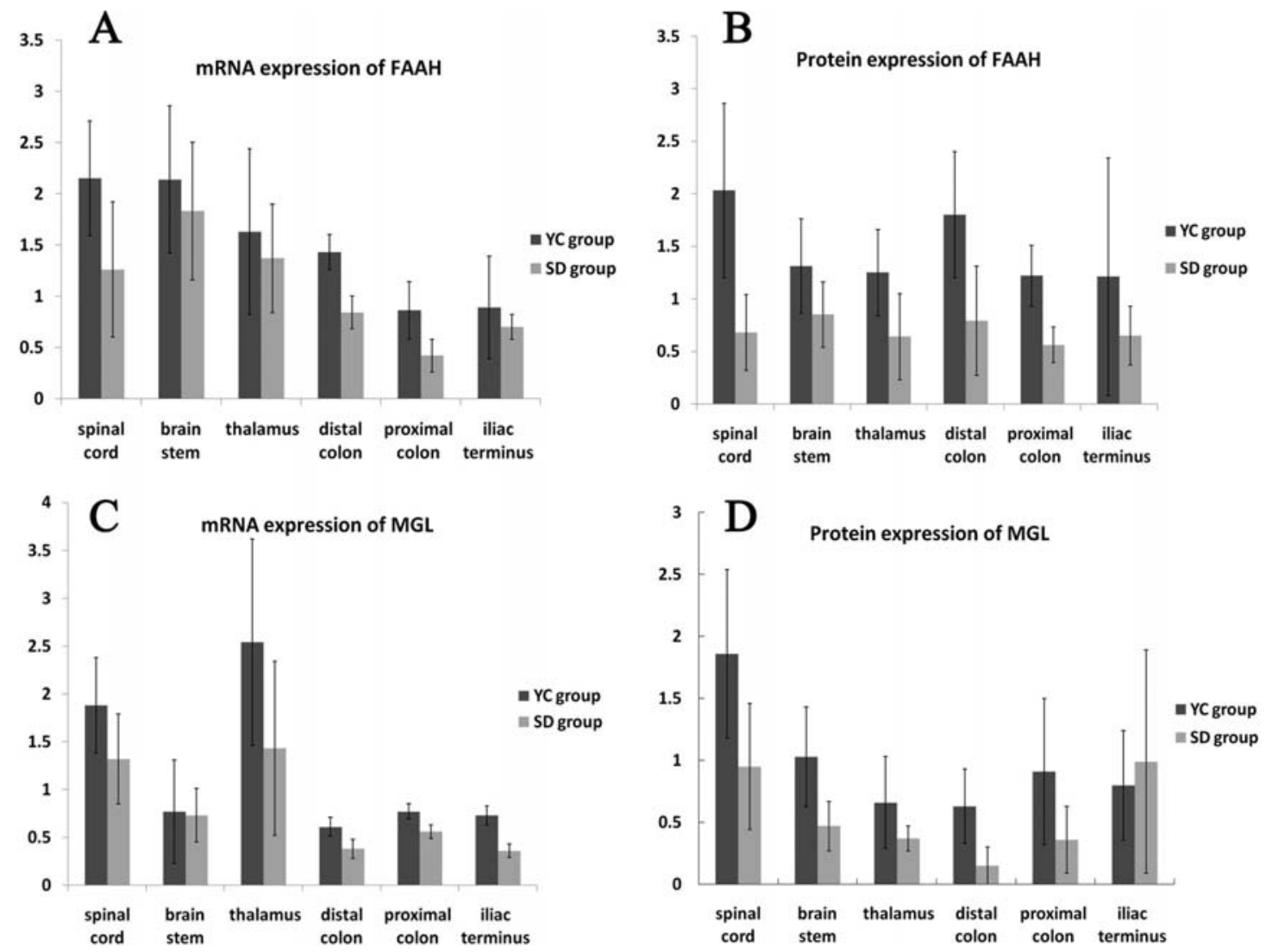

Figure 4. Quantity alteration in mRNA and protein expressions of FAAH and MGL in each region after REMSD for $48 \mathrm{~h}$. (A) Differences in mRNA expression of FAAH between the SD and control group in the CNS and gut (P-value was $0.008,0.56,0.27,0.002,0.016$ and 0.247 in spinal cord, brain stem, thalamus, distal and proximal colon and iliac terminus, respectively). (B) Differences in protein expression of FAAH between the SD and control group in the CNS and gut (P-value was $0.001,0.031,0.01,0.02,0.037$ and 0.093 in spinal cord, brain stem, thalamus, distal and proximal colon and iliac terminus, respectively). (C) Differences in mRNA expression of MGL between the SD and control group in the CNS and gut (P-value was $0.037,0.85,0.043,0.001$, 0.002 and 0.151 in spinal cord, brain stem, thalamus, distal and proximal colon and iliac terminus, respectively). (D) Differences in protein expression of MGL between the SD and control group in the CNS and gut (P-value was 0.04, 0.004, 0.026, 0.047, 0.017 and 0.364 in spinal cord, brain stem, thalamus, distal and proximal colon and iliac terminus, respectively). SD group, REMSD group. 

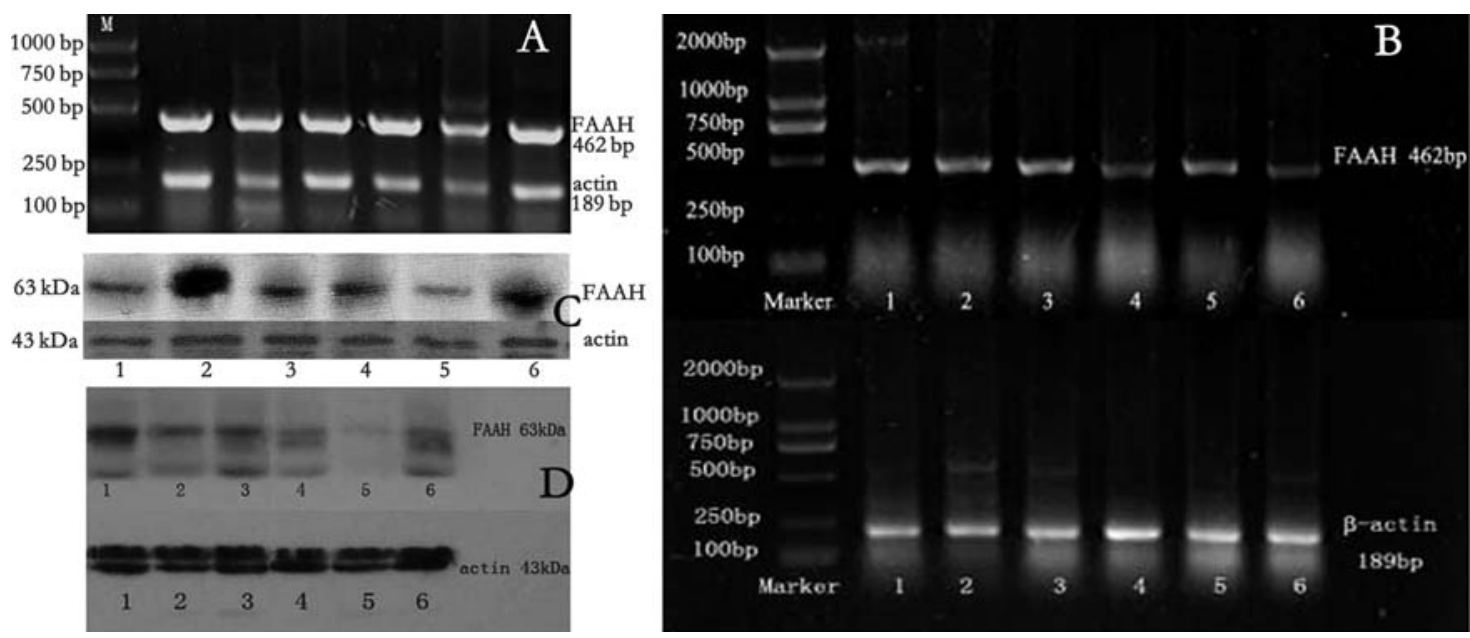

Figure 5. Difference in mRNA (A and B) and protein (C and D) expressions of FAAH in each region after REMSD for $48 \mathrm{~h}$. (A) mRNA expression of FAAH in the CNS region. Lane 1, spinal cord of REMSD; 2, spinal cord of YC; 3, brain stem of REMSD; 4, brain stem of YC; 5, thalamus of REMSD; and 6, thalamus of YC group. (B) mRNA expression of FAAH in the gut. Lane 1, distal colon of YC; 2, distal colon of REMSD; 3, proximal colon of YC; 4, proximal colon of REMSD; 5, iliac terminus of YC; and 6, iliac terminus of REMSD group. (C) Protein expression of FAAH in the CNS region. Lane 1, spinal cord of REMSD; 2, spinal cord of YC; 3, brain stem of REMSD; 4, brain stem of YC; 5, thalamus of REMSD and 6, thalamus of YC group. (D) Protein expression of FAAH in the gut. Lane 1, distal colon of YC; 2, iliac terminus of YC; 3, proximal colon of YC; 4, distal colon of REMSD; 5, iliac terminus of REMSD; and 6, proximal colon of REMSD group.

was then transferred to polyvinylidene ethylene membranes, blocked from the non-specific antibody binding sites with nonfat milk powder (concentration of $0.5 \%$ ) at room temperature for $2 \mathrm{~h}$, and then polyclonal antibodies (1:100) against CB1R (Abcam), FAAH (Chemicon), and MGL (Abcam) were added. This was followed by incubation overnight at $4^{\circ} \mathrm{C}$, then incubation with a secondary antibody (1:5000) for $2 \mathrm{~h}$, and then autoradiograms were taken with ECL reagent in the chamber. Finally, imaging analysis was carried out by an automated electrophoresis gel imaging system.

Data presentation and statistical analysis. We used SPSS 12.0 statistical software for the analysis, the data were displayed as the means \pm SEM, and Pearson's correlation analysis was used to analyze the correlation of the discharge frequency of the external oblique muscle under different pressure conditions. In the cases of statistical significance, statistical single-factor analyses of the discharge frequencies of the three groups, were carried out by variance analysis. The differences within each group as well as those between groups, were analyzed. If significance of variance was found, an independent samples t-test was used to summarize the different data. An independent samples t-test was also used in the analysis of the expression of RNA and protein. A value of $\mathrm{P}<0.05$ was considered to be significant.

\section{Results}

There is a close relationship between distension pressure and discharge frequency of the external oblique muscle. In order to ensure the reliability of the experiments, we analyzed the CRD and EMG activity of the abdominal wall of all the rats prior to the experiment. The results showed that, with increasing pressure, the contraction strength of the superior oblique abdominal wall was enhanced, and simultaneously the discharge frequency increased. Discharge frequencies under the pressure of $20,40,60$ and $80 \mathrm{mmHg}$ were (239.63 \pm 98.32$),(592.87 \pm 250.25),(1373.55 \pm 374.28)$ and (1809.96 \pm 386.57$)$, respectively. The data demonstrate that the discharge frequency was closely related to the visceral sensitivity.

REMSD decreases the abdominal wall EMG activity of the rats, and Rimonabant intervention antagonizes that effect in the REMSD rats. Before REMSD, the discharge frequencies of the external oblique muscle under each pressure condition were not significantly different among the three groups (Fig. 1A). However, after REMSD for $48 \mathrm{~h}$, there was a statistically significant difference between the two groups. In the group with the Rimonabant intervention after REMSD for $48 \mathrm{~h}$, the responses to CRD were significantly increased compared to those in the REMSD group, which showed an increase in discharges of the abdominal oblique muscle. There was no significant difference between the Rimonabant and the control group (Fig. 1B). The results suggest that Rimonabant antagonizes the visceral hyposensitivity induced by REMSD.

REMSD enhances $m R N A$ expression of CBIR, in both the $C N S$ and peripheral gut, but increased protein expression is only observed in CNS region after REMSD. After REMSD for $48 \mathrm{~h}$, the RNA expression of CB1R in all the regions (thalamus, brain stem, spinal cord, proximal and distal colon, and the iliac terminus) was higher than that in the corresponding control group (Figs. 2A, 3A and B). While the enhanced protein expressions in the REMSD group compared to the control group were consistent with the RNA expression in the CNS, we could not find a significant difference in the protein expression between the REMSD and control groups in the gut region (Figs. 2B, 3C and D). 

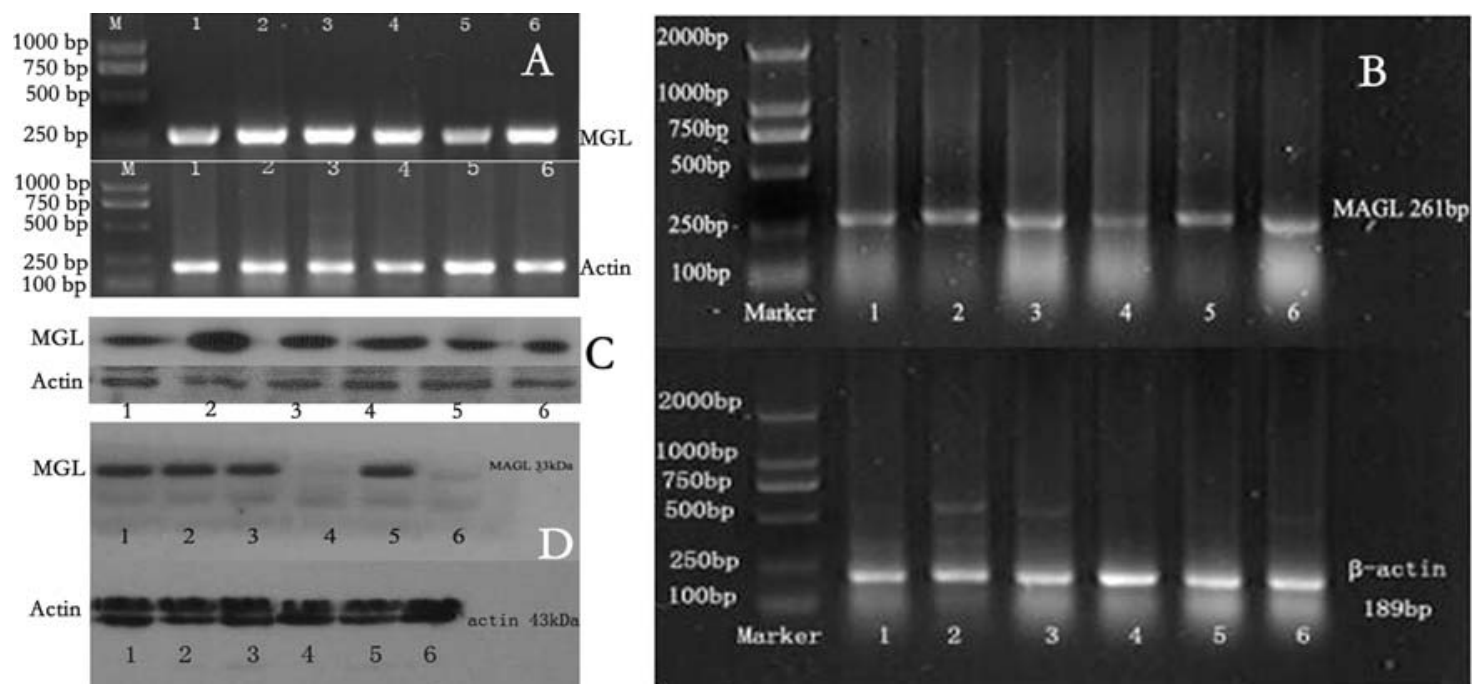

Figure 6. Difference in mRNA (A and B) and protein (C and D) expressions of MGL in each region after REMSD for $48 \mathrm{~h}$. (A) mRNA expression of MGL in the CNS region. Lane 1, spinal cord of REMSD; 2, spinal cord of YC; 3, brain stem of REMSD; 4, brain stem of YC; 5, thalamus of REMSD; and 6, thalamus of YC group. (B) mRNA expression of MGL in the gut. Lane 1, distal colon of YC; 2, distal colon of REMSD; 3, proximal colon of YC; 4, proximal colon of REMSD; 5, iliac terminus of YC; and 6, iliac terminus of REMSD group. (C) Protein expression of MGL in the CNS region. Lane 1, spinal cord of REMSD; 2, spinal cord of YC; 3, brain stem of REMSD; 4, brain stem of YC; 5, thalamus of REMSD; and 6, thalamus of YC group. (D) Protein expression of MGL in the gut. Lane 1, distal colon of YC; 2, iliac terminus of YC; 3, proximal colon of YC; 4, distal colon of REMSD; 5, iliac terminus of REMSD; and 6, proximal colon of REMSD group.

Influence of REMSD on the expression levels of FAAH and $M G L$ in the CNS and peripheral gut. After REMSD for $48 \mathrm{~h}$, the levels of FAAH and MGL RNA expression in the thalamus, spinal cord and colon were lower in the REMSD compared to the control group. However, no difference was found in other regions such as the brain stem and the iliac terminus (Figs. 4A and C, 5A and B and 6A and B). At the same time, we observed that the protein expressions in all CNS regions examined as well as in the colon, were lower in the REMSD compared to the control group. However, again no significant difference was found in the iliac terminus (Figs. 4B and D, 5C and D and 6C and D). Therefore, we consider the synthesis of FAAH and MGL in the CNS and colon to be decreased after REMSD.

\section{Discussion}

Our present study demonstrates that REMSD induces visceral hyposensitivity in rats, and that the intervention with the $\mathrm{CB}$ receptor antagonist, Rimonabant, prevents this effect. In addition, we provide a direct comparison of differently altered endocannabinoid receptor expressions and their hydrolases in the CNS and peripheral gut regions, and show that the expression of CB1R is significantly increased after REMSD in the CNS region, but not in the peripheral gut region, while the expressions of the hydrolases are significantly decreased both in the CNS and the colon, but not in the iliac terminus.

Based on the principle that visceral pain induces a reflex muscle contraction, we recorded the cluster discharge and behavioral observations when the muscle of the abdominal wall contracted, to reflect the visceral sensitivity in rats. We found a significant reduction in the number of discharges of the abdominal oblique muscle in the sleep-deprived rats when exposed to different pressure conditions, which is consistent with results from our prior studies (3). Other studies have also shown a reduction in pain perception in rats that are deprived of REM sleep (21). Therefore, we further confirm that after REMSD, rats show decreased responses to injurious and non-injurious stimuli, exhibiting a decline in visceral sensitivity.

Furthermore, in our study, Rimonabant had an antagonistic effect on the visceral hyposensitivity induced by REMSD. This finding indicates that endocannabinoids are possibly associated with the decreased visceral sensitivity induced by REMSD. Endocannabinoids have previously been reported to be effective in persistent pain states, by inhibiting hyperalgesia in models of chronic neuropathic (22) or inflammatory (23-25) pain. However, this is the first study using models of visceral sensitivity. Many previous studies have shown that endogenous $\mathrm{CBs}$ produce antinociception through CB1R and that Rimonabant antagonizes the sensory-decreasing effects of the cannabinoids by blocking CB1R (26-28). Therefore, it can be speculated that REMSD plays a role in the regulation of visceral sensitivity through the endogenous $\mathrm{CB}$-mediated mechanism. However, it is not clear which areas (the brain or gut) play a role in this endocannabinoid effect.

After $48 \mathrm{~h}$ of REMSD, the expressions of both FAAH and MGL significantly decreased in the CNS and colon, but not in the iliac terminus, thus suggesting that REMSD can induce the decrease in FAAH and MGL levels in these regions. This finding is consistent with ones from previous studies, as the inhibition of the hydrolytic enzymes of endocannabinoids, FAAH and MGL, enhances the content of endocannabinoids and prolongs their effect. Kathuria et al have indicated that the FAAH inhibitor, URB597, significantly enhances the levels of anandamide in the brain (29). In addition, Capasso et al found that the content of intestinal anandamide in FAAH gene knockout mice was 2.8 -fold higher than that in 
wild-type mice (30). Castelli et al treated rats with the CB receptor agonist, and then found increased $\mathrm{CB} 1 \mathrm{R}$ expression in the brain, which was accompanied by the decreased expressions of FAAH and MGL. They postulated that this was due to the increased driving force activity of endogenous CBs, or an adaptive response of the body (31). Our results show that there is no significant change in the gene and protein expressions of FAAH and MGL in the iliac terminus after REMSD. Capasso et al showed that the expression of FAAH mRNA gradually decreased in the distal and proximal colon as well as the ileum, due to the low expression of FAAH in the ileum under physiological conditions. It is considered that REMSD cannot have a significant impact on the expression of FAAH and MGL, and that endocannabinoids in the ileum do not have a significant effect on the visceral sensitivity decreased by REMSD.

We also found that the increased protein expression of CB1R in the CNS but not the gut, is also associated with the visceral hyposensitivity induced by REMSD, and that sleep deprivation leads to the gene and protein expression of the endogenous CB1R in the CNS region. This finding is in agreement with previous data showing that the increased gene expression of $\mathrm{CB} 1 \mathrm{R}$ in the hippocampus and brain stem, is found after short-term REMSD (15). After $48 \mathrm{~h}$ of continuous sleep deprivation, through a CB1R-mediated mechanism, the enhanced endogenous CBs act on the increased CB1R in the CNS and produce a similar effect as the cannabinoid receptor agonists. Neuroanatomical studies have shown that, the CB1R, the endogenous cannabis-like type, as well as the degradation enzymes of endogenous CBs, are mainly located in the CNS, especially in the regions that are important for the transduction and regulation of pain signals, such as amygdala, thalamus and midbrain gray matter (32). In the spine, CB1R is located in the intrinsic neurons and sensory afferent nerve terminals of the spinal cord (33). Endogenous $\mathrm{CBs}$ in the midbrain and spinal cord produce a non-opioiddependent analgesic effect by means of the CB1R-mediated pathway $(34,35)$. In addition, it has been proven that the well established antinociceptive activity of CBs in animal models of acute pain, is mediated via the activation of supraspinal and spinal CB1R (36). In our study, the altered expression of CB1R after REMSD is mainly found in the CNS but not in the gut. Therefore, it is reasonable to believe, that the influence of REMSD on visceral sensitivity is closely related with the increased $\mathrm{CB} 1 \mathrm{R}$ expression in the CNS region.

In summary, we conclude that, the endocannabinoid system is involved in the mechanism of rat visceral hyposensitivity induced by REMSD. However, it displays different actions in the brain and gut regions. There was an enhanced concentration of endocannabinoids in both the brain and gut, which was due to the reduction in their hydrolases. However, the enhanced expression of CBIR was only found in the brain. Perhaps the CNS region plays the main role in the endocannabinoid activity.

\section{Acknowledgements}

This study was carried out in a laboratory in the Division of Gastroenterology Laboratory, Union Hospital, Wuhan, China. We thank all the colleagues in this laboratory for their assistance, and especially Jinping Zhang, for his advice in this study.

\section{References}

1. De Ponti F and Malagelada JR: Functional gut disorders: from motility to sensitivity disorders. A review of current and investigational drugs for their management. Pharmacol Ther 80 : 49-88, 1998.

2. Giedke H, Klingberg S, Schwarzler F and Schweinsberg M: Direct comparison of total sleep deprivation and late partial sleep deprivation in the treatment of major depression. J Affect Disord 76: 85-93, 2003.

3. Wang LY, Yang T, Qian W and Hou XH: [Effects of central endocannabinoid system on visceral hyposensitivity induced by rapid eye movement sleep deprivation: experiment with rats] Zhonghua Yi Xue Za Zhi 89: 559-563, 2009

4. Piomelli D: The molecular logic of endocannabinoid signalling. Nat Rev Neurosci 4: 873-884, 2003

5. Palazzo E, Marabese I, de Novellis V, et al: Metabotropic and NMDA glutamate receptors participate in the cannabinoidinduced antinociception. Neuropharmacology 40: 319-326, 2001.

6. Howlett AC, Barth F, Bonner TI, et al: International Union of Pharmacology. XXVII. Classification of cannabinoid receptors. Pharmacol Rev 54: 161-202, 2002.

7. Hama A and Sagen J: Antinociceptive effect of cannabinoid agonist WIN 55,212-2 in rats with a spinal cord injury. Exp Neurol 204: 454-457, 2007.

8. Hohmann AG: Spinal and peripheral mechanisms of cannabinoid antinociception: behavioral, neurophysiological and neuroanatomical perspectives. Chem Phys Lipids 121: 173-190, 2002.

9. Derbenev AV, Stuart TC and Smith BN: Cannabinoids suppress synaptic input to neurones of the rat dorsal motor nucleus of the vagus nerve. J Physiol 559: 923-938, 2004.

10. Deutsch DG and Chin SA: Enzymatic synthesis and degradation of anandamide, a cannabinoid receptor agonist. Biochem Pharmacol 46: 791-796, 1993.

11. Dinh TP, Kathuria S and Piomelli D: RNA interference suggests a primary role for monoacylglycerol lipase in the degradation of the endocannabinoid 2-arachidonoylglycerol. Mol Pharmacol 66: 1260-1264, 2004.

12. Chen C and Bazan NG: Lipid signaling: sleep, synaptic plasticity, and neuroprotection. Prostaglandins Other Lipid Mediat 77: 65-76, 2005.

13. Huitron-Resendiz S, Sanchez-Alavez M, Wills DN, Cravatt BF and Henriksen SJ: Characterization of the sleep-wake patterns in mice lacking fatty acid amide hydrolase. Sleep 27: 857-865, 2004.

14. Martinez-Vargas M, Murillo-Rodriguez E, Gonzalez-Rivera R, et al: Sleep modulates cannabinoid receptor 1 expression in the pons of rats. Neuroscience 117: 197-201, 2003.

15. Jiang PF, Zhu T and Xia ZZ: [Cannabinoid receptor 1 expression and pathological changes in rat hippocampus after deprivation of rapid eye movement sleep]. Zhejiang Da Xue Xue Bao Yi Xue Ban 35: 535-540, 2006.

16. Hornby PJ and Prouty SM: Involvement of cannabinoid receptors in gut motility and visceral perception. Br J Pharmacol 141: 1335-1345, 2004.

17. Sanson M, Bueno L and Fioramonti J: Involvement of cannabinoid receptors in inflammatory hypersensitivity to colonic distension in rats. Neurogastroenterol Motil 18: 949-956, 2006.

18. Benedito MA and Camarini R: Rapid eye movement sleep deprivation induces an increase in acetylcholinesterase activity in discrete rat brain regions. Braz J Med Biol Res 34: 103-109, 2001.

19. Liang LX, Zhang Q, Qian W and Hou XH: Antinociceptive property of tegaserod in a rat model of chronic visceral hypersensitivity. Chin J Dig Dis 6: 21-25, 2005.

20. Al-Chaer ED, Kawasaki M and Pasricha PJ: A new model of chronic visceral hypersensitivity in adult rats induced by colon irritation during postnatal development. Gastroenterology 119 : 1276-1285, 2000.

21. Hakki Onen S, Alloui A, Jourdan D, Eschalier A and Dubray C: Effects of rapid eye movement (REM) sleep deprivation on pain sensitivity in the rat. Brain Res 900: 261-267, 2001.

22. Herzberg U, Eliav E, Bennett GJ and Kopin IJ: The analgesic effects of R(+)-WIN 55,212-2 mesylate, a high affinity cannabinoid agonist, in a rat model of neuropathic pain. Neurosci Lett 221: 157-160, 1997. 
23. Clayton N, Marshall FH, Bountra C and O'Shaughnessy CT: $\mathrm{CB} 1$ and $\mathrm{CB} 2$ cannabinoid receptors are implicated in inflammatory pain. Pain 96: 253-260, 2002

24. Jaggar SI, Hasnie FS, Sellaturay S and Rice AS: The antihyperalgesic actions of the cannabinoid anandamide and the putative CB2 receptor agonist palmitoylethanolamide in visceral and somatic inflammatory pain. Pain 76: 189-199, 1998.

25. Martin WJ, Loo CM and Basbaum AI: Spinal cannabinoids are anti-allodynic in rats with persistent inflammation. Pain 82: 199-205, 1999

26. Martin BR and Lichtman AH: Cannabinoid transmission and pain perception. Neurobiol Dis 5: 447-461, 1998

27. Pertwee RG: Cannabinoid receptors and pain. Prog Neurobiol 63: 569-611, 2001

28. Pertwee RG, Gibson TM, Stevenson LA, et al: O-1057, a potent water-soluble cannabinoid receptor agonist with antinociceptive properties. Br J Pharmacol 129: 1577-1584, 2000.

29. Kathuria S, Gaetani S, Fegley D, et al: Modulation of anxiety through blockade of anandamide hydrolysis. Nat Med 9: 76-81, 2003.

30. Capasso R, Matias I, Lutz B, et al: Fatty acid amide hydrolase controls mouse intestinal motility in vivo. Gastroenterology 129: 941-951, 2005.
31. Castelli MP, Paola Piras A, D'Agostino A, et al: Dysregulation of the endogenous cannabinoid system in adult rats prenatally treated with the cannabinoid agonist WIN 55,212-2. Eur J Pharmacol 573: 11-19, 2007.

32. Martin WJ, Coffin PO, Attias E, Balinsky M, Tsou K and Walker JM: Anatomical basis for cannabinoid-induced antinociception as revealed by intracerebral microinjections. Brain Res 822: 237-242, 1999.

33. Hohmann AG, Briley EM and Herkenham M: Pre- and postsynaptic distribution of cannabinoid and mu opioid receptors in rat spinal cord. Brain Res 822: 17-25, 1999.

34. Fox A, Kesingland A, Gentry C, et al: The role of central and peripheral Cannabinoid1 receptors in the antihyperalgesic activity of cannabinoids in a model of neuropathic pain. Pain 92: 91-100, 2001.

35. Hohmann AG, Suplita RL, Bolton NM, et al: An endocannabinoid mechanism for stress-induced analgesia. Nature 435: 1108-1112, 2005

36. Dyson A, Peacock M, Chen A, et al: Antihyperalgesic properties of the cannabinoid CT-3 in chronic neuropathic and inflammatory pain states in the rat. Pain 116: 129-137, 2005. 